\title{
Comparisons Among Multiple Machine Learning Based Classifiers for Breast Cancer Risk Stratification Using Electrical Impedance Spectroscopy
}

\author{
Md. Toukir Ahmed, Md. Rayhanul Masud and Abdullah Al Mamun
}

\begin{abstract}
Nowadays, women worldwide are affected greatly by breast cancer. The consequences of the disease and the number of affected are so alarming that it requires immediate attention. Prediction of the disease is the primary and most significant route to prevention of it. This study aims to have a comparison among multiple machine learning based classifiers for breast cancer risk stratification with the help of resonancefrequency electrical impedance spectroscopy. Five machine learning based classifiers namely- Naïve Bayes, Multilayer perceptron, J48, Bagging and Random Forest were applied to the dataset and a comparison was made based on different performance metrics. The study demonstrated that Random Forest classifier performed slightly better than the others in both splitting and folding of the dataset.
\end{abstract}

Index Terms - Breast Cancer, Classifier, Machine Learning, Spectroscopy.

\section{INTRODUCTION AND LITERATURE REVIEW}

Breast cancer is the most common malignancy among women worldwide and it is the second leading cause of women death. Breast cancer screening is a significant step for early detection and to guarantee a greater possibility of having a good result in treatment [1]. It is crucial to discover prognostic factors and therapeutic targets to fight the disease [2]. Well-built risk factors for the development and progression of this kind of tumors are prolonged lifespan [3]. Epidemiological studies support that obesity is positively connected with the continuance of different types of cancer, namely breast, and also with increased cancer mortality [3]. The rate of death is decreasing day by day because of finding latent cause of breast cancer, earlier diagnosis, increased awareness. The health and medical sectors nowadays are relying on data mining which helps medical practitioners to extract valuable information from large database to have a clear conception that may assist for future treatment or research. For breast cancer data mining can be very effective contributing to medication and rectifying hospital data errors. In many developed and developing country breast cancer is very common and second leading cause of dead. In US and in

Published on July 18, 2020

Md. Toukir Ahmed, Pabna University of Science and Technology, Bangladesh.

(corresponding e-mail: toukirahmedreal@ gmail.com)

Md. Rayhanul Masud, Bangladesh University of Engineering and Technology, Bangladesh.

(e-mail: r.masudsaom ${ }^{\circledR}$ gmail.com)
Asia the cause of death in breast cancer is high level. Among Asian countries breast cancer is very common in Pakistan approximately 90000 [4].

Breast cancer is typically diagnosed using a tissue biopsy. Sample tissue is collected from the suspected patient which is used for histological and chemical analysis.

It usually needs 1-2 days to get result. Moreover, sometimes patients require to go for biopsy test again due to undecided results. If tissue analysis could be done faster or some methodologies could be discovered to determine the candidacy of a sample of tissue to be used for further screening, stress of the patients waiting for the results and the cost of screening, both could be lessened. Complex impedance properties of a material is measured with the help of a method, called Electrical Impedance Spectroscopy (EIS). The system is feed a voltage or current of known frequency and amplitude and the resulting current or voltage is recorded.

The differences of phase and magnitude between applied stimulus and response determine the complex impedance at that frequency. The changes of the complex impedance of the system with stimulus frequency is determined through repeating the process for a range of frequencies. In recent decades, electric and di-electric measurements have been carried out in breast tissue under a range of experimental conditions [5].

Robust predictive models relied on data, collected in routine consultation and blood analysis contributes by offering more screening tools [1]. Several candidates for predictors of breast cancer have been reported in the literature [6]. A prediction model for breast cancer patients' pathologic response before neoadjuvant chemotherapy was invented [7]. The predictors were tumor haemoglobin parameters generated by ultrasound-guided near-infrared optical tomography in association with standard pathologic tumor characteristics [7]. Several authors gave importance to assess the risk of breast cancer [8]. Finally, artificial intelligence and machine learning techniques were imposed on databases and made publicly available in the UCI Machine Learning Repository. In particular, there has been an astounding number of work published on the Wisconsin Breast Cancer Dataset (WBCD), the Wisconsin Diagnosis Breast Cancer (WDBC) and the Wisconsin Prognosis Breast Cancer

Abdullah Al Mamun, Bangladesh University of Engineering and Technology, Bangladesh

(e-mail: kamrul1203@gmail.com) 
(WPBC) $[9,10]$. In the same order, they provide cytology data which can be used for differentiating malignant from benign samples, features computed from a digitized image of a fine needle aspirate of a breast mass again used for classifying as malignant or benign and follow-up data for breast cancer patients that can be used to predict cancer recurrence [1]. There are two types of breast cancer treatment which are local and systematical. Local types of treatments are surgery and radiation. Chemotherapy and hormone therapy are systematical type of treatment. Medical practitioners continue both types of treatment collaterally for checking which one impacting best.

Owing to the drawbacks of available imaging-based screening tools, interest in developing new approaches for risk assessment of breast cancer has increased. The primary idea is to develop simple, easy to implement, inexpensive tools for stratifying women into two groups namely, those who are at "average or low risk" and those who are at significantly "higher-than-average" risk of having or developing breast cancer. Women categorized in the highrisk group would then be recommended for imaging-based follow-up [11]. The models used in this work are based on a population with early-diagnosed breast cancer. In this paper, different data classification algorithms are used which is described in Method section and the used dataset is dataset with electrical impedance measurements in samples of freshly excised tissue from the breast [5]. First of all, a superior classification algorithm has been found out among used classifiers compared with accuracy and other standard parameters, then the dataset is analyzed to extract the feature of attributes effectiveness and based on that the dataset is modified. After that the best performing classifier is run on the modified dataset for getting the improved performances result. The description of the data collected and statistical methods used in the article are presented on the Methods section. The results are then discussed on a separate section and finally the main conclusions are drawn.

\section{Methodology}

This section is divided into several subsections. First a short description about dataset is discussed, then a brief notes on some machine learning algorithms is addressed and finally the performance metrics are also discussed shortly which are used to evaluate models.

\section{A. Dataset}

The dataset used in this study has been extracted from UCI Machine Learning Repository [5]. Clinical features used in the dataset were observed or measured for 106 instances. There are 9 predictors, all quantitative, and a dependent variable, indicating one of the six classes of the examined breast tissue. Prediction models based on these predictors, if accurate, can potentially be used as a biomarker of breast cancer. The attribute list used in the Breast Cancer Dataset is listed in TABLE I.

\section{B. Used Classifiers}

Naïve Bayes is a probabilistic statistical base classifier based upon Bayes' theorem which is strong supervised machine learning classification technique. It assumes that all the features are conditionally independent [12] which means the effect of an attribute value has no effect on other attribute value. Naïve Bayes is a very light weight classifier which can be used to classify big dataset easily. It is very robust to ignore noise and irrelevant attributes. It is very easy to construct and has no need of complicated iterative parameter estimation scheme [13].

TABLE I: ATTRIBUTES INFORMATION OF DATASET WITH ELECTRICAL IMPEDENCE MEASUREMENTS IN SAMPLES OF FRESHLY EXCISED TISSUE FROM THE BREAST

\begin{tabular}{|c|c|}
\hline Attributes Name & Attribute Value Type \\
\hline Impedivity (ohm) at zero frequency (I0) & quantitative \\
\hline phase angle at $500 \mathrm{KHz}(\mathrm{PA} 500)$ & quantitative \\
\hline high-frequency slope of phase angle (HFS) & quantitative \\
\hline $\begin{array}{l}\text { impedance distance between spectral ends } \\
\text { (DA) }\end{array}$ & quantitative \\
\hline area under spectrum (AREA) & quantitative \\
\hline area normalized by DA (A/DA) & quantitative \\
\hline maximum of the spectrum (Max IP) & quantitative \\
\hline $\begin{array}{l}\text { distance between } \mathrm{I} 0 \text { and real part of the } \\
\text { maximum frequency point (DR) }\end{array}$ & quantitative \\
\hline length of the spectral curve $(\mathrm{P})$ & quantitative \\
\hline $\begin{array}{l}\text { Six classes of freshly excised tissue were } \\
\text { studied using electrical impedance } \\
\text { Carcinoma (Car), } \\
\text { Fibro-adenoma (Fad), } \\
\text { Mastopathy (Mas), } \\
\text { Glandular (Gla), } \\
\text { Connective(Con), Adipose(Adi) } \\
\end{array}$ & Class variable \\
\hline
\end{tabular}

Multilayer perceptron (MLP) belongs to one of the classes of neural network and a branch of artificial intelligence [14]. It is a kind of acyclic graph and comes under the category of supervised feed forward networks. MLP has three or more layers of nodes such as input layer, hidden layer and output layer, each node is a neuron which uses non-linear activation function except for the input node. It connects multiple layers in a directed graph to establish one way directed signal path through the nodes and each node has a non-linear activation function. It can generalize new unseen data easily and the core feature of multilayer perceptron is, it doesn't make any prior assumption regarding data ordering.

Random forest is a supervised learning method which is a decision tree based algorithm. As the name suggests as forest the random forest classifier is an ensemble of decision trees where a random vector sample produces each classifier from input vector [15] and each tree casts a unit vote for the most popular class to classify an input vector, most of the time trained with a bagging method. The general idea of bagging method is that a composition of learning method increases the overall result. The Random Forest shows less sensitivity to overfitting and the quality of training samples than any other mainstream machine learning classifiers. Bagging predictor generates several versions of a predictor which are used to derive an aggregated predictor. When a numerical outcome is predicted, the aggregation averages over the versions. The aggregation illustrates a plurality voting when a class is predicted. These multiple versions are formed from the 
replication of the learning set which are used as new learning sets in this formulation.

Bagging provides substantial gains in case of accuracy when real and simulated data sets are tested using classification and regression trees and subset selection in linear regression where instability is the key element of the prediction method. Its accuracy can be improved if significant changes are made in the constructed predictor through perturbing the learning set [16].

\section{Performance Metrics}

Performance parameters are the most important metrics to compare among classifier methods to get the best classifier. We have applied 10 performance parameters which are Accuracy, Sensitivity, Specificity, Kappa statistics, Precision, Recall, F-measure, Matthew's Correlation Coefficient (MCC), Receiver Operating Characteristic (ROC) Area, Precision Recall Curves (PRC). These parameters are calculated from a confusion matrix which is situated in every step of classification.

TP represents the number of correctly classified positive instances.

FP represents the number of misclassified positive instances. FN represents the number of misclassified negative instances. TN represents the number of correctly classified negative instances.

$$
\begin{aligned}
& \text { Accuracy }=\frac{T P+T N}{T P+T N+F P+F N} \\
& \text { Specificity }=\frac{T N}{T N+F P} \\
& \text { Sensitivity }=\frac{T P}{T P+F N} \\
& \text { Precision }=\frac{T P}{T P+F P} \\
& \text { Recall }=\frac{T P}{T P+F N} \\
& \text { F-measure }=\frac{2 * \text { precision } * \text { recall }}{\text { precision }+ \text { recall }}
\end{aligned}
$$

MCC $=$ measure of quality of binary classification.

ROC Area $=$ Most important parameter in WEKA which defines the classifier's performance in general.

PRC area $=$ This is also an important parameter which is more informative, a plot of precision and recall. This is very useful to get the difference between precision and recall.

\section{IMPLEMENTATION}

In this section we discuss about our proposed methodology which we apply step by step to get expected result. WEKA (Waikato Environment for Knowledge Analysis) [17] is a powerful tool as it contains supervised learning as well as unsupervised learning methods. It contains classification, clustering, association, mining, feature selection, data visualization etc. Weka is a very useful data mining tool that helps researchers to implement classification, analyze data, visualize data, comparing classification techniques to get easily the better performing algorithm using various parameters. At first, we analyze the dataset in WEKA data mining tool 3.8 which is used to analyze and has various techniques embedded in it. Performance of the proposed classifiers is assessed and better performing classifier is identified. Best performing classifier is selected based on not only accuracy but also other metrics as well.

\section{RESUlTS AND DISCUSSION}

In this section, results of the study is discussed. Five different classifier algorithms were imposed on the dataset and based on different performance metrics, their result is compared and the best performing classifier for this particular dataset is found out. Firstly, the dataset is divided into two parts: $66.67 \%$ of the data for training and then $33.33 \%$ remaining for test. The result of this splitting can be found in bar chart (Fig. 1.) and in curve (Fig. 2.).

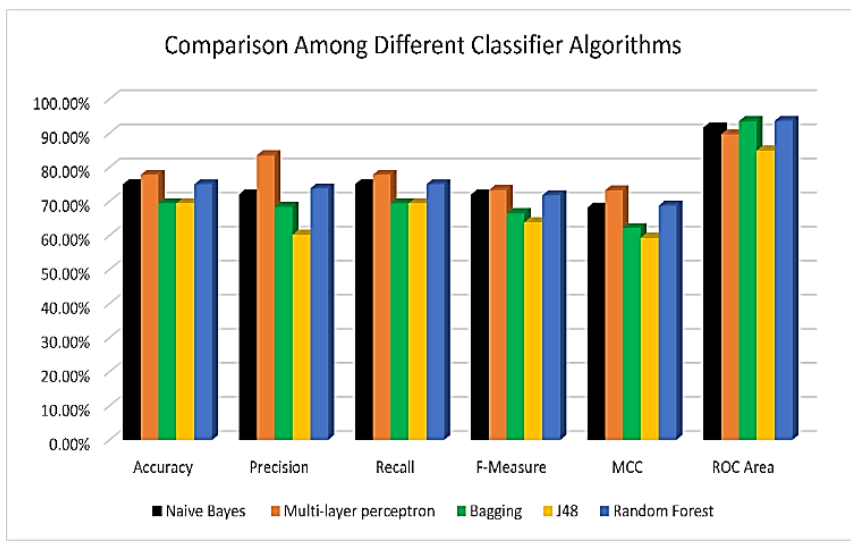

Fig. 1. Bar Chart showing performance of different Classifier Using $66.67 \%-33.33 \%$ split on Dataset

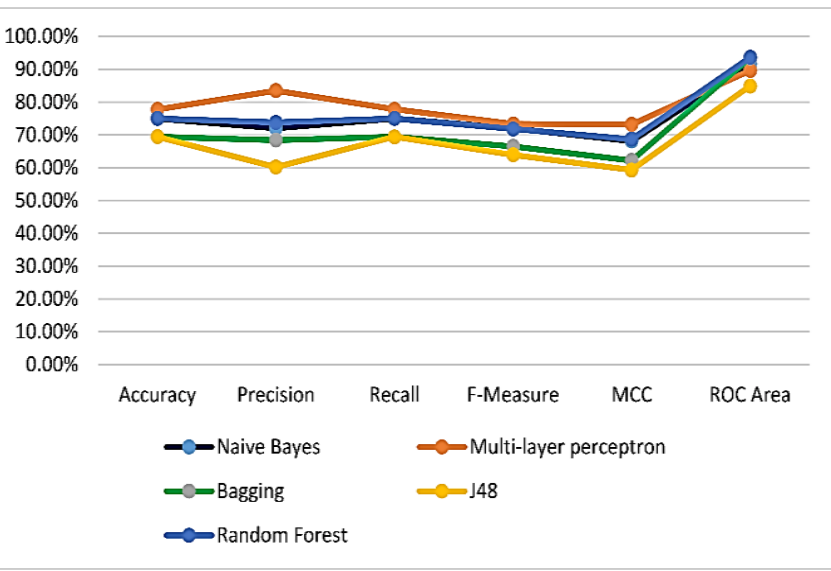

Fig. 2. Line Chart showing performance of different Classifier Using $66.67 \%-33.33 \%$ split on Dataset

From Fig. 1. and Fig. 2., it is evident that Multi-Layer Perceptron classifier works better than others based on all the performance metrics used for $66.67 \%-33.33 \%$ splitting on the dataset.

Apart from splitting, 10-Fold cross-validation was also used to have a proper testing on the dataset as well. The result of the classifiers after 10-fold is listed graphically in Fig. 3 . and Fig 4. 


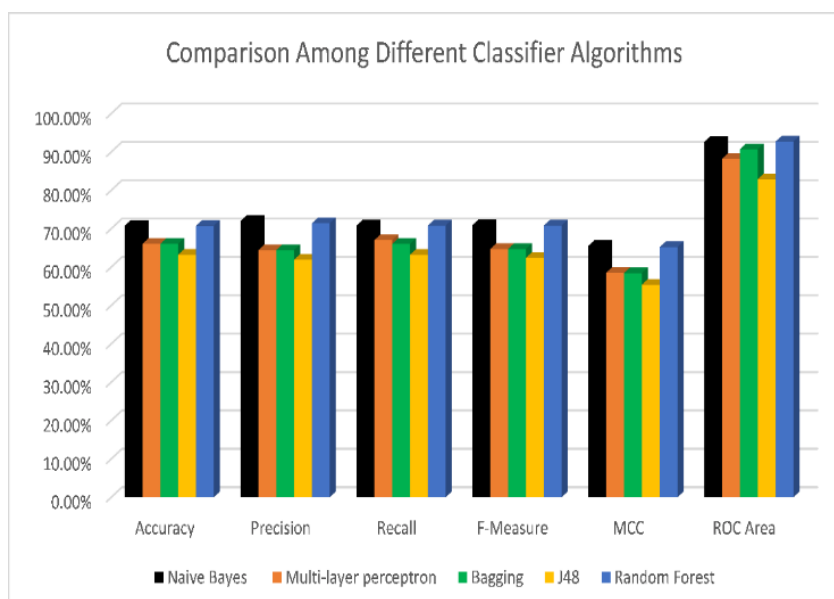

Fig. 3. Bar Chart showing performance of different Classifier Using 10-fold cross validation on Dataset

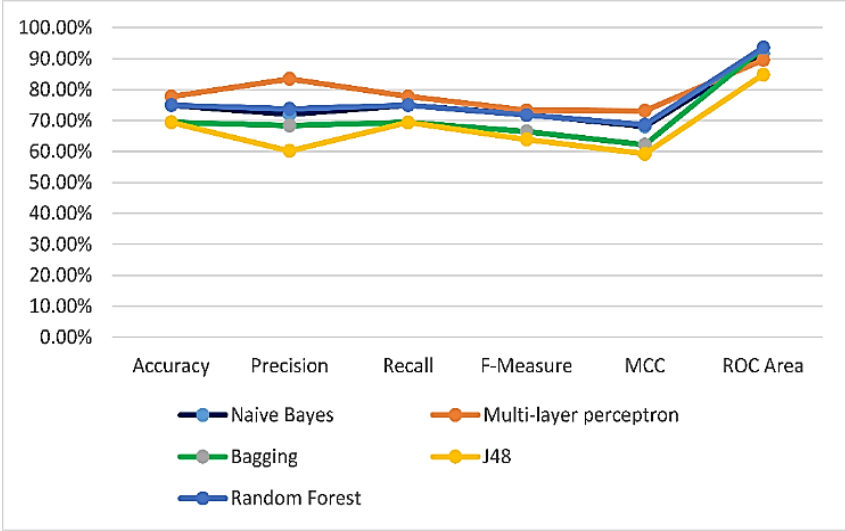

Fig. 4. Line Chart showing performance of different Classifier Using 10fold cross validation on Dataset

From Fig. 3. and Fig. 4., it is evident that Multi-layer perceptron performs better than other classifiers after 10 fold cross validation too. So, we conclude our result section having Multi-Layer Perceptron as top performing classifier among the classifiers used in the study though it is also noticeable that Random Forest classifier performs better in terms of ROC Area only.

\section{CONCLUSION AND FUTURE DiRECTION}

Breast cancer being one of the malignancies worldwide, needs to be identified and treated accurately and timely. In this work, we have used five classification algorithms and come to a point that Multi-Layer Perceptron is superior than others compared with standard parameters. After that, analysis of the dataset to extract the features of attributes to know the effectiveness of different attributes was also done with no such noticeable improvements in the result. But leading to such normal accuracy, it has made us think whether the dataset was oversaturated. So, in future, we will try to find out the oversaturation of the reformed dataset and run a Principal Component Analysis (PCA) on the dataset.

\section{REFERENCES}

[1] M. P. J. C. J. M. P. G. M. S. R. a. C. F. Patrício, "Using Resistin glucose, age and BMI to predict the presence of breast cancer.," BMC cancer, vol. 18(1), p. 29, 2018.
[2] J. M. P. S.-S. D. G. A. G. M. P. M. L. L. S.-R. A. S. L. a. S. R. Crisóstomo, " Hyperresistinemia and metabolic dysregulation: a risky crosstalk in obese breast cancer.," Endocrine, vol. 53(2), pp. 433-442, 2016.

[3] M. Dalamaga, "Obesity, insulin resistance, adipocytokines and breast cancer: New biomarkers and attractive therapeutic targets," World journal of experimental medicine, vol. 3(3), p. 34, 2013.

[4] Malik, "Clinico-pathological features of breast cancer in Pakistan," JOURNAL-PAKISTAN MEDICAL ASSOCIATION, vol. 52(3), pp. 100-103, 2002.

[5] J. M. d. S. J. J. JE Silva, "Classification of Breast Tissue by Electrical Impedance Spectroscopy," Med \& Bio Eng \& Computing, vol. 38, pp. 26-30., 2000.

[6] K. H. H. a. W. L. Cole, "Breast cancer biomarker measurements and standards," PROTEOMICS-Clinical Applications, Vols. 7(1-2), pp. 17-29, 2013.

[7] F. a. M. K. Xue, "Diabetes, metabolic syndrome, and breast cancer: a review of the current evidence," The American journal of clinical nutrition, vol. 86(3), pp. 823S-835S, 2007.

[8] Y. C. X. Q. M. C. P. T. H. H. Y. a. H. Y. Huang, "Prediabetes and the risk of cancer: a meta-analysis," Diabetologia, vol. 57, no. 11, pp. 2261 2269, 2014.

[9] R. Ahima, "Adipose tissue as an endocrine organ. Obesity," vol. 14, no. S8, pp. 242S-249S, 2006.

[10] C. W. M. C. C. C. Y. H. G. Y. T. C. W. Y. C. a. Y. J. Sun, "Adipocytokine resistin and breast cancer risk," Breast cancer research and treatment, vol. 123, no. 3, pp. 869-876, 2010.

[11] D. Z. B. W. X. W. X. H. \&. G. D. Lederman, "Improving breast cancer risk stratification using resonance-frequency electrical impedance spectroscopy through fusion of multiple classifiers.," Annals of biomedical engineering, vol. 39, no. 3, pp. 931-945., 2011.

[12] D. Parkin, "Epidemiology of cancer: global patterns and trends," Toxicology Letters, Vols. 102-103, pp. 227-234, Dec 1998.

[13] K. Xindong Wu, "The Top Ten Algorithms in Data Mining," Knowl Inf Syst , pp. 1-37, 2014.

[14] M. \&. D. S. Gardner, "Artificial neural networks (the multilayer perceptron) - a review of applications in the atmospheric sciences," Atmospheric Environment, vol. 32, no. 14, pp. 2627 - 2636, 1998.

[15] L. D. Mariana Belgiu, "Random forest in remote sensing: A review of applications and future directions," Journal ArticlePhotogrammetry and Remote Sensing, vol. 114, pp. 24-31, Apr 2016

[16] L. Breiman, "Bagging predictors," Machine learning,, vol. 24, no. 2, pp. 123-140, 1996

[17] E. F. G. H. B. P. P. R. I. H. W. Mark Hall, "The WEKA data mining software," ACM SIGKDD Explorations Newsletter, vol. 11, no. 1, p. 10, 16 Nov 2009.

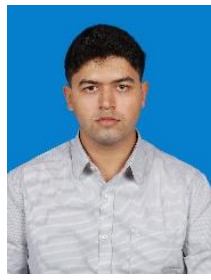

Md. Toukir Ahmed received his B.Sc. Engineering in Computer Science and Engineering from Bangladesh University of Engineering and Technology (BUET), Dhaka, Bangladesh in 2017. He is currently working as a Lecturer in the Department of Computer Science and Engineering, Pabna University of Science and Technology, Pabna, Bangladesh. His research interest includes Data Mining, HCI, Big Data Analysis and Machine Learning.

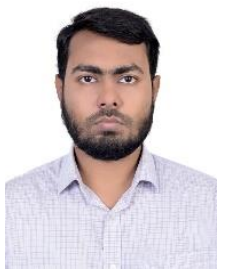

Md. Rayhanul Masud received his B.Sc Engineering in Computer Science and Engineering from Bangladesh University of Engineering an Technology (BUET), Dhaka, Bangladesh in 2017. He is currently working as an Engineer at Kona Software Lab Limited, Dhaka, Bangladesh. His research interest includes Distributed Systems, Blockchain and Machine Learning.

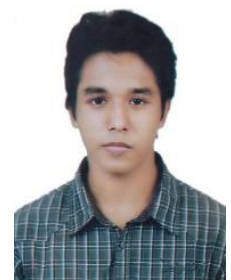

Abdullah Al Mamun received his B.Sc. Engineering in Computer Science and Engineering from Bangladesh University of Engineering an Technology (BUET), Dhaka, Bangladesh in 2017. He is currently working as a Software Engineer at Divergent Technologies, Dhaka, Bangladesh. His research interest includes Data Mining, HCI, Big Data Analysis and Machine Learning. 